\title{
Using Spinning Dust Emission To Constrain The Evolution Of Dust Grains In Cold Clumps
}

\author{
C.T. Tibbs $*{ }^{a}$ R. Paladini, ${ }^{a}$ K. Cleary, ${ }^{a}$ K.J.B. Grainge, ${ }^{b}$ S.J.C. Muchovej, ${ }^{a}$ T.J. \\ Pearson, ${ }^{a}$ Y.C. Perrott, ${ }^{c}$ C. Rumsey, ${ }^{c}$ A.M.M. Scaife, ${ }^{d}$ M.A. Stevenson ${ }^{a}$ and J. \\ Villadsen ${ }^{a}$ \\ ${ }^{a}$ California Institute of Technology, Pasadena, CA 91125, USA \\ ${ }^{b}$ University of Manchester, Manchester, M13 9PL, UK \\ ${ }^{c}$ University of Cambridge, Cambridge, CBE OHE, UK \\ ${ }^{d}$ University of Southampton, Southampton, SO17 1BJ, UK \\ E-mail: ctibbsaipac.caltech.edu, paladinieipac.caltech.edu, \\ kcleary@astro.caltech.edu, keith.grainge@manchester.ac.uk, \\ sjcmeastro.caltech.edu, tjp@astro.caltech.edu, \\ ycp21@mrao.cam.ac.uk, cr461@mrao.cam.ac.uk, a.scaife@soton.ac.uk, \\ maseastro.caltech.edu, jrveastro.caltech.edu
}

\begin{abstract}
Within many molecular clouds in our Galaxy there are cold, dense regions known as cold clumps in which stars form. These dense environments provide a great location in which to study dust grain evolution. Given the low temperatures $(\sim 10-15 \mathrm{~K})$ and high densities $\left(\sim 10^{5} \mathrm{~cm}^{-3}\right)$, these environments are dark at mid-infrared (IR) wavelengths and emit strongly at wavelengths $\geq 160 \mu \mathrm{m}$. The lack of mid-IR emission can be attributed to one of two reasons: i) a deficit of the small dust grains that emit stochastically at mid-IR wavelengths; or ii) small dust grains are present, but due to the high densities, the stellar photons cannot penetrate deep enough into the clumps to excite them. Using mid-IR observations alone it is impossible to distinguish between these two scenarios. However, by using spinning dust emission at $\mathrm{cm}$ wavelengths it is possible to break this degeneracy, because if small dust grains are present in these clumps, then even though stellar photons cannot excite them to emit at mid-IR wavelengths, these dust grains will be spunup by collisions and hence emit spinning dust radiation. If spinning dust were detected in these clumps it would prove that there are small dust grains present and that the lack of mid-IR emission is due to a lack of stellar photons. Conversely, a lack of spinning dust emission would indicate a deficit of small dust grains in these clumps. Since small dust grains require harsh radiation fields to be destroyed, a lack of small dust grains is likely a result of dust grain coagulation. With this in mind, we present preliminary results illustrating our method of using spinning dust observations to determine the evolution of small dust grains in these environments.
\end{abstract}

The Life Cycle of Dust in the Universe: Observations, Theory, and Laboratory Experiments 18-22 November, 2013

Taipei, Taiwan

${ }^{*}$ Speaker. 


\section{Introduction}

Star formation is the result of gravitational instability occurring in cold, dense structures known as pre-stellar cores. These cores represent one of the earliest phases of star formation and the investigation of the physical properties of these environments is crucial for understanding the early stages of star formation. These dense environments also play a vital role in the life cycle of dust in the Galactic interstellar medium, making them an ideal location in which to study dust grain evolution. As part of the Planck Early Release Compact Source Catalog [1], the Early Cold Cores Catalog (ECC) containing all of the Galactic cold clumps ${ }^{1}$ with temperatures $<14 \mathrm{~K}$ and a signal to noise ratio $>15$ was published. Recent modelling [2] found that if there are small dust grains present in these clumps then spinning dust emission should be detected. Spinning dust emission is a relatively new emission mechanism due to electric dipole emission from small, spinning dust grains [3]. Observed in the wavelength range $3-0.3 \mathrm{~cm}$, spinning dust emission has been detected in a variety of Galactic environments (e.g. $[4,5,6,7])$, and produces a very distinctive peaked spectrum, peaking at wavelengths of $\sim 1 \mathrm{~cm}$. Given this result [2], spinning dust observations can be used to probe the abundance of the small dust grains, and hence the dust grain evolution, in these dense environments. To test this hypothesis, we observed 15 of the Planck ECC sources with the CARMA interferometer at $1 \mathrm{~cm}$. In this analysis we simply focus on one of our targets, ECC224, which is located in the Cepheus molecular cloud complex at $l=113.62^{\circ}, b=+15.01^{\circ}$. Displayed in Figure 1 are maps of ECC224 from mid-infrared (IR) to $\mathrm{cm}$ wavelengths. The emission is most prominent in the far-IR and sub-mm wavelengths, with little or no emission at shorter (70 and $100 \mu \mathrm{m})$ and longer $(1 \mathrm{~cm})$ wavelengths. The lack of emission at 70 and $100 \mu \mathrm{m}$ is due to the fact that this clump is cold, while the lack of emission at $1 \mathrm{~cm}$, implies that there is no significant spinning dust emission. However, even with a no detectable spinning dust emission, it is still possible to constrain the evolution of dust grains in this clump.

\section{Thermal Dust Emission}

To model the thermal dust emission in ECC224 we used Herschel data. All of the Herschel maps were reprocessed, convolved to a common angular resolution, and to remove any contribution from foreground/background emission, we subtracted the median value of the flux computed in a reference position that is devoid of emission in each of the maps. We modelled the far-IR emission using $I_{v}=\mu m_{\mathrm{H}} N_{\mathrm{H}} \kappa_{v} B_{v}\left(T_{\mathrm{d}}\right)$, where $I_{v}$ is the intensity at frequency $v, \mu$ is the molecular weight of hydrogen, $m_{\mathrm{H}}$ is the mass of a $\mathrm{H}$ atom, $N_{\mathrm{H}}$ is the hydrogen column density, $B_{v}\left(T_{\mathrm{d}}\right)$ is the Planck function for temperature $T_{\mathrm{d}}$, and $\kappa_{v}$ is the dust opacity which was assumed to be of the form: $\kappa_{v} \propto v^{\beta}$, with $\beta=2$. Given the lack of emission at 70 and $100 \mu \mathrm{m}$, we excluded these bands and fitted the 160, 250, 350, and $500 \mu \mathrm{m}$ maps on a pixel by pixel basis for $N_{\mathrm{H}}$ and $T_{\mathrm{d}}$ (see Figure 2).

Herschel, with its sub-arcmin angular resolution, is able to resolve the structure in the Planck ECC sources (see Figure 1) and for this reason, we used CLUMPFIND [8] to identify sub-clumps in ECC224 (see Figure 2). Since CLUMPFIND computes the effective circular radius of each subclump, we assumed that the sub-clumps are spherical. For each sub-clump we computed the mean column density, $N_{\mathrm{H}}^{\text {mean }}$, and mean dust temperature, $T_{\mathrm{d}}^{\text {mean }}$. We divided $N_{\mathrm{H}}^{\text {mean }}$ by the linear size of

\footnotetext{
${ }^{1}$ We refer to the Planck sources as clumps as Planck lacks the angular resolution to observe individual cores.
} 

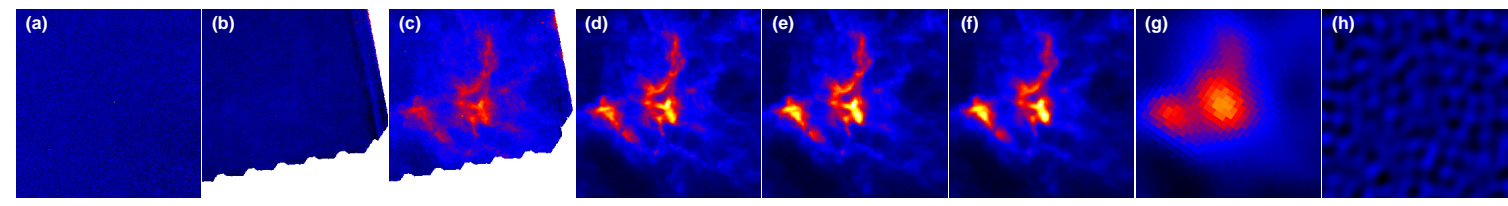

Figure 1: Maps of ECC224: (a) Herschel $70 \mu \mathrm{m}$ map; (b) Herschel $100 \mu \mathrm{m}$ map; (c) Herschel $160 \mu \mathrm{m}$ map; (d) Herschel $250 \mu \mathrm{m}$ map; (e) Herschel $350 \mu \mathrm{m}$ map; (f) Herschel $500 \mu \mathrm{m}$ map; (g) Planck $850 \mu \mathrm{m}$ map; (h) CARMA $1 \mathrm{~cm}$ map. All maps are $30 \operatorname{arcmin} \times 30$ arcmin in size and centered on ECC224.
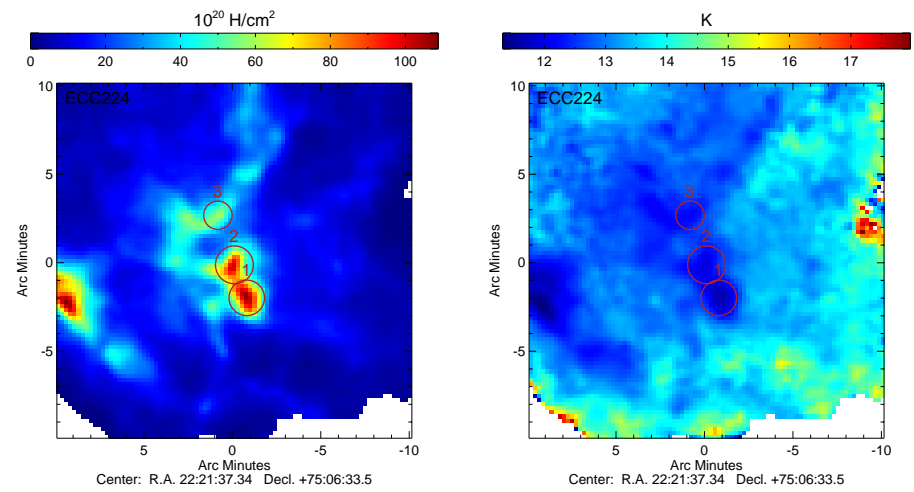

Figure 2: $N_{\mathrm{H}}$ and $T_{\mathrm{d}}$ maps of ECC224 derived from the Herschel observations with the three identified sub-clumps labelled.

the sub-clumps to estimate the mean density, $n_{\mathrm{H}}$, and we converted $T_{\mathrm{d}}^{\text {mean }}$ into the mean radiation field, $\chi$, using $\chi=\left(T_{\mathrm{d}} / 17.5\right)^{(4+\beta)}$.

\section{Spinning Dust Emission}

To estimate the expected level of spinning dust emission in ECC224 we used the spinning dust model SPDUST [9] with $n_{\mathrm{H}}$ and $\chi$ (derived from the Herschel observations), in combination with the idealized parameters for dark clouds [3], as input parameters. The dust grain size distribution incorporated within SPDUST is parametrized using the ratio of total to selective extinction, $R_{\mathrm{V}}$, and the abundance of the small carbonaceous dust grains, $\mathrm{b}_{\mathrm{C}}$ [10]. Using the $R_{\mathrm{V}}=5.5$ size distributions, we computed the spinning dust emission for four different values of $b_{\mathrm{C}}$. The final SEDs are plotted in Figure 3, with the far-IR emission constrained using the Herschel data (asterisks), and the predicted spinning dust curves plotted at microwave frequencies. It is clear to see how the amplitude of the spinning dust curves increases as the abundance of the small carbonaceous dust grains increases. Given the lack of detection, we plotted the conservative $5 \sigma$ upper limit estimated from the $1 \mathrm{~cm}$ CARMA data (solid triangle), which allows us to constrain the abundance of the small carbonaceous dust grains by ruling out values of $b_{C}>2 \times 10^{-5}$ for each of the sub-clumps.

\section{Conclusions}

The goal of this work is to demonstrate the ability of using spinning dust observations to determine how dust grains are evolving in cold clumps. We use Herschel far-IR observations of one of the Planck cold clumps (ECC224) to derive maps of $N_{\mathrm{H}}$ and $T_{\mathrm{d}}$, which we use to estimate $n_{\mathrm{H}}$ and $\chi$ in identified sub-clumps. These derived properties are used as inputs to the spinning 

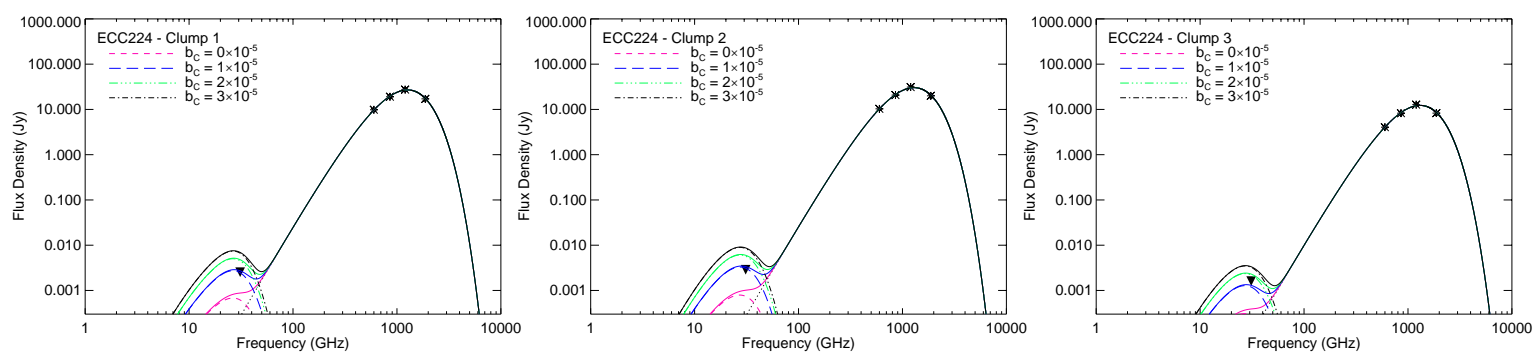

Figure 3: SEDs of the three sub-clumps identified in ECC224. The spinning dust curves are produced using SPDUST based on $n_{\mathrm{H}}$ and $\chi$ derived from the far-IR emission for a range of different values of the abundance of small carbonaceous dust grains $\left(\mathrm{b}_{\mathrm{C}}\right)$. The $1 \mathrm{~cm}$ data point (solid triangle) is the $5 \sigma$ upper limit estimated from the CARMA data. Also plotted are the flux densities of the Herschel data (asterisks) at 160, 250, 350, and $500 \mu \mathrm{m}$.

dust model SPDUST to predict the level of spinning dust emission for each of the sub-clumps, for a range of abundances of the small carbonaceous dust grains. We compare the predicted spinning dust emission with CARMA $1 \mathrm{~cm}$ observations to constrain the small dust grain abundance. We find that for the three sub-clumps in ECC224, there is no detectable emission at $1 \mathrm{~cm}$, and that the three sub-clumps exhibit a deficit of small dust grains, suggesting that dust grain coagulation is occurring. This is the first time that spinning dust observations have been used to constrain the evolutionary properties of dust grains.

\section{References}

[1] Planck Collaboration, Planck early results. VII. The Early Release Compact Source Catalogue, 2011, A\&A, 536, A7

[2] N. Ysard, M. Juvela, \& L. Verstraete, Modelling the spinning dust emission from dense interstellar clouds, 2011, A\&A, 535, A89

[3] B.T. Draine, \& A. Lazarian, Electric Dipole Radiation from Spinning Dust Grains, 1998, ApJ, 508, 157

[4] Planck Collaboration, Planck intermediate results. XV. A study of anomalous microwave emission in Galactic clouds, 2013, arXiv:1309.1357

[5] C.T. Tibbs, R.A. Watson, C. Dickinson, et al., Very Small Array observations of the anomalous microwave emission in the Perseus region, 2010, MNRAS, 402, 1969

[6] C. Dickinson, S. Casassus, R.D. Davies, et al., Infrared-correlated 31-GHz radio emission from Orion East, 2010, MNRAS, 407, 2223

[7] C.T. Tibbs, R. Paladini, M. Compiègne, et al., A Multi-wavelength Investigation of RCW175: An H II Region Harboring Spinning Dust Emission, 2012, ApJ, 754, 94

[8] J.P. Williams, E.J. de Geus, \& L. Blitz, Determining structure in molecular clouds, 1994, ApJ, 428, 693

[9] K. Silsbee, Y. Ali-Haïmoud, \& C.M. Hirata, Spinning dust emission: the effect of rotation around a non-principal axis, 2011, MNRAS, 411, 2750

[10] J.C. Weingartner, \& B.T. Draine, Dust Grain-Size Distributions and Extinction in the Milky Way, Large Magellanic Cloud, and Small Magellanic Cloud, 2001, ApJ, 548, 296 